Supplement of Biogeosciences, 15, 703-719, 2018

https://doi.org/10.5194/bg-15-703-2018-supplement

(C) Author(s) 2018. This work is distributed under

the Creative Commons Attribution 3.0 License.

(c) (1)

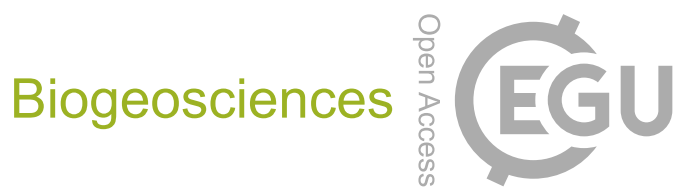

Supplement of

\title{
Peat decomposability in managed organic soils in relation to land use, organic matter composition and temperature
}

Cédric Bader et al.

Correspondence to: Jens Leifeld (jens.leifeld@agroscope.admin.ch)

The copyright of individual parts of the supplement might differ from the CC BY 3.0 License. 
Table S1

Tab. S 1: Mean and standard errors of the bulk density, SOC content, $\mathrm{C} / \mathrm{N}, \mathrm{H} / \mathrm{C}$ and $\mathrm{O} / \mathrm{C}$ ratio as well as $\mathrm{CO}_{2}$ emissions $\left(\mathrm{mg} \mathrm{CO}_{2}-\mathrm{C}\right.$ g SOC$\left.{ }^{-1}\right)$ at 10 and $20^{\circ} \mathrm{C}$. In addition, we displayed the inclinations derived from the robust regression made for every sample after incubation.

\begin{tabular}{|c|c|c|c|c|c|c|c|c|c|c|c|c|c|c|c|c|c|c|c|}
\hline & \multirow{2}{*}{\begin{tabular}{|l|}
$\begin{array}{l}\text { Depth } \\
(\mathrm{cm})\end{array}$ \\
\end{tabular}} & \multicolumn{2}{|c|}{$\mathrm{BD}\left(\mathrm{g} \mathrm{cm}^{-3}\right)$} & \multicolumn{2}{|c|}{ SOC $\left(\mathrm{g} \mathrm{kg}^{-1}\right)$} & \multicolumn{2}{|c|}{$\mathrm{C} / \mathrm{N}$ ratio } & \multicolumn{2}{|c|}{$\mathrm{H} / \mathrm{C}$ ratio } & \multicolumn{2}{|c|}{$\mathrm{O} / \mathrm{C}$ ratio } & \multicolumn{2}{|c|}{$\mathrm{CO}_{2}$ at $10^{\circ} \mathrm{C}$} & \multicolumn{2}{|c|}{ Inclination at $10^{\circ} \mathrm{C}$} & \multicolumn{2}{|c|}{$\mathrm{CO}_{2}$ at $20^{\circ} \mathrm{C}$} & \multicolumn{2}{|c|}{ Inclination at $20^{\circ} \mathrm{C}$} \\
\hline & & mean & SE & mean & SE & mean & SE & mean & SE & mean & SE & mean & SE & mean & SE & mean & $\mathrm{SE}$ & mean & SE \\
\hline \multirow{6}{*}{$\overrightarrow{0}$} & $0-10$ & 0.729 & 0.060 & 252.28 & 22.82 & 14.03 & 0.44 & 1.44 & 0.02 & 0.60 & 0.05 & 190.0 & 1.8 & $-1.52 \times 10^{-1}$ & $2.00 \times 10^{-2}$ & 44.8 & 2.9 & $-1.56 \times 10^{-1}$ & $6.79 \times 10^{-2}$ \\
\hline & $10-20$ & 0.888 & 0.085 & 278.46 & 9.91 & 13.98 & 0.33 & 1.45 & 0.03 & 0.55 & 0.04 & 19.3 & 1.5 & $-1.78 \times 10^{-1}$ & $5.77 \times 10^{-2}$ & 47.0 & 3.3 & $-1.76 \times 10^{-1}$ & $2.99 \times 10^{-2}$ \\
\hline & $20-30$ & 0.873 & 0.234 & 271.48 & 14.07 & 11.48 & 3.03 & 1.49 & NA & 0.60 & NA & 17.0 & NA & $-6.41 \times 10^{-2}$ & NA & 41.0 & NA & $-7.56 \times 10^{-2}$ & NA \\
\hline & $30-40$ & 1.158 & 0.095 & 243.58 & 18.90 & 13.06 & 0.93 & 1.46 & 0.04 & 0.62 & 0.02 & 18.3 & 1.9 & $-2.11 \times 10$ & $3.85 \times 10^{-2}$ & 42.3 & 1.8 & $-1.46 \times 10^{-1}$ & $7.03 \times 10^{-3}$ \\
\hline & $40-50$ & 0.560 & - & 337.40 & - & 16.08 & - & & & & & & & & & & & & \\
\hline & $50-60$ & 0.650 & - & 313.60 & - & 16.50 & - & 1.38 & NA & 0.62 & NA & 57.0 & NA & $-2.72 \times 10^{-1}$ & NA & 75.0 & NA & $-2.40 \times 10-1$ & NA \\
\hline \multirow{8}{*}{ ज) } & $0-10$ & 0.304 & 0.033 & 244.91 & 14.07 & 13.56 & 0.25 & 1.44 & 0.04 & 0.67 & 0.03 & 23.5 & 3 & $-1.50 \times 10^{-1}$ & $7.86 \times 10^{-2}$ & 48.3 & 6.4 & $-1.12 \times 10^{-1}$ & $2.71 \times 10^{-2}$ \\
\hline & $10-20$ & 0.334 & 0.015 & 246.08 & 13.45 & 13.27 & 0.28 & 1.47 & 0.02 & 0.67 & 0.02 & 20.5 & 2.4 & $-2.17 \times 10^{-1}$ & $5.28 \times 10^{-2}$ & 46.0 & 2.2 & $-7.49 \times 10^{-2}$ & $1.09 \times 10^{-1}$ \\
\hline & $20-30$ & 0.364 & 0.043 & 252.55 & 12.65 & 13.52 & 0.40 & 1.38 & 0.03 & 0.65 & 0.05 & 19.0 & 2 & $-1.89 \times 10^{-1}$ & $6.90 \times 10^{-2}$ & 50.5 & 4.5 & $-1.64 \times 10^{-1}$ & $4.43 \times 10^{-3}$ \\
\hline & $30-40$ & 0.226 & 0.034 & 245.17 & 13.90 & 13.59 & 0.28 & 1.43 & 0.04 & 0.60 & 0.01 & 21.0 & & $-3.86 \times 10^{-2}$ & $5.25 \times 10^{-2}$ & 52.5 & 14.5 & $-2.11 \times 10^{-1}$ & $9.09 \times 10^{-2}$ \\
\hline & $40-50$ & 0.278 & 0.095 & 241.21 & 9.29 & 14.12 & 0.52 & 1.34 & NA & 0.59 & NA & 38.5 & 3.5 & $-2.37 \times 10^{-1}$ & $4.54 \times 10^{-2}$ & 62.0 & NA & $-2.68 \times 10^{-1}$ & NA \\
\hline & $50-60$ & 0.277 & 0.052 & 335.00 & 62.30 & 15.73 & 2.74 & & & & & & & & & & & & \\
\hline & $60-70$ & 0.340 & - & - & - & - & - & & & & & & & & & & & & \\
\hline & $70-80$ & 0.400 & - & - & - & - & - & & & & & & & & & & & & \\
\hline \multirow{5}{*}{ 可 } & $0-10$ & 0.538 & 0.036 & 196.19 & 12.79 & 12.11 & 0.13 & 1.59 & 0.04 & 0.68 & 0.06 & NA & NA & NA & NA & 74.3 & 11.6 & $-2.33 \times 10-1$ & $8.27 \times 10^{-2}$ \\
\hline & $10-20$ & 0.649 & 0.035 & 192.43 & 12.74 & 12.40 & 0.14 & 1.73 & NA & 0.39 & NA & 29.7 & 4.8 & $-2.05 \times 10^{-1}$ & $7.64 \times 10^{-2}$ & 93.0 & NA & $-4.42 \times 10^{-1}$ & NA \\
\hline & $20-30$ & 0.758 & 0.070 & 263.48 & 35.99 & 14.63 & 1.08 & 1.43 & 0.03 & 0.49 & 0.05 & 27.7 & 0.7 & $-1.99 \times 10^{-1}$ & $4.47 \times 10^{-2}$ & 61.7 & 3.8 & $-1.87 \times 10^{-1}$ & $3.80 \times 10^{-2}$ \\
\hline & $30-40$ & 0.743 & 0.197 & 266.65 & 39.66 & 14.58 & 0.81 & & & & & & & & & & & & \\
\hline & $40-50$ & 0.690 & - & 211.30 & - & 14.02 & - & & & & & & & & & & & & \\
\hline \multirow{8}{*}{$\begin{array}{l}0 \\
0\end{array}$} & $0-10$ & 0.486 & 0.032 & 160.68 & 10.70 & 12.53 & 0.81 & 1.67 & 0.02 & 0.70 & 0.07 & 30.3 & 2.1 & $-1.70 \times 10^{-1}$ & $2.81 \times 10^{-2}$ & 59.3 & 3.4 & $-1.82 \times 10^{-1}$ & $2.40 \times 10^{-2}$ \\
\hline & $10-20$ & 0.610 & 0.029 & 164.02 & 2.77 & 12.88 & 0.18 & 1.66 & 0.04 & 0.73 & 0.05 & 27.5 & 1.8 & $-1.44 \times 10^{-1}$ & $2.06 \times 10^{-2}$ & 51.0 & 3.3 & $-1.99 \times 10^{-1}$ & $2.80 \times 10^{-2}$ \\
\hline & $20-30$ & 0.523 & 0.044 & 166.14 & 4.07 & 12.82 & 0.14 & 1.64 & 0.01 & 0.68 & 0.05 & 25.3 & 1.8 & $-1.35 \times 10^{-1}$ & $2.82 \times 10^{-2}$ & 54.5 & 2.4 & $-1.34 \times 10^{-1}$ & $1.13 \times 10^{-2}$ \\
\hline & $30-40$ & 0.356 & 0.023 & 199.13 & 9.19 & 14.02 & 0.36 & 1.54 & 0.04 & 0.59 & 0.02 & 33.8 & 1.9 & $-2.29 \times 10^{-1}$ & $4.20 \times 10^{-2}$ & 49.8 & 2.6 & $-1.50 \times 10^{-1}$ & $2.28 \times 10^{-2}$ \\
\hline & $40-50$ & 0.224 & 0.024 & 322.59 & 20.88 & 16.24 & 0.69 & 1.43 & 0.05 & 0.55 & 0.04 & 27.3 & 2 & $-1.51 \times 10^{-1}$ & $6.26 \times 10^{-2}$ & 66.0 & 11.6 & $-6.51 \times 10^{-2}$ & $7.23 \times 10^{-3}$ \\
\hline & $50-60$ & 0.230 & 0.036 & 297.92 & 28.21 & 15.91 & 0.52 & 1.55 & 0.15 & 0.70 & 0.07 & 25.0 & 2 & $-7.12 \times 10^{-2}$ & $3.32 \times 10^{-2}$ & 50.0 & 1 & $-1.17 \times 10^{-1}$ & $2.77 \times 10^{-2}$ \\
\hline & $60-70$ & 0.225 & 0.092 & 265.17 & 69.58 & 14.58 & 0.77 & & & & & & & & & & & & \\
\hline & $70-80$ & 0.075 & 0.005 & 321.62 & 28.58 & 17.37 & 0.67 & & & & & & & & & & & & \\
\hline & $0-10$ & 0.566 & 0.028 & 111.05 & 2.07 & 12.96 & 0.22 & 1.66 & 0.07 & 0.44 & 0.04 & 25.0 & 4.6 & $-2.21 \times 10^{-1}$ & $6.67 \times 10^{-2}$ & 55.0 & 12.2 & $-2.04 \times 10^{-1}$ & $4.37 \times 10^{-2}$ \\
\hline & $10-20$ & 0.725 & 0.028 & 99.21 & 3.45 & 12.67 & 0.27 & 1.67 & 0.03 & 0.51 & 0.05 & 29.3 & 3.7 & $-1.59 \times 10^{-1}$ & $3.18 \times 10^{-2}$ & 48.5 & 6 & $-2.48 \times 10^{-1}$ & $4.41 \times 10^{-2}$ \\
\hline & $20-30$ & 0.676 & 0.056 & 99.99 & 2.45 & 12.61 & 0.19 & 1.62 & 0.05 & 0.49 & 0.04 & 25.8 & 1.1 & $-2.07 \times 10^{-1}$ & $1.28 \times 10^{-2}$ & 53.0 & 1.4 & $-1.84 \times 10^{-1}$ & $1.79 \times 10^{-2}$ \\
\hline$\overline{0}$ & $30-40$ & 0.536 & 0.023 & 128.00 & 12.16 & 12.68 & 0.36 & 1.67 & 0.11 & 0.38 & 0.11 & 34.3 & 2 & $-2.26 \times 10^{-1}$ & $4.09 \times 10^{-2}$ & 52.0 & 2 & $-1.53 \times 10-1$ & $2.01 \times 10^{-2}$ \\
\hline$\cup$ & $40-50$ & 0.506 & 0.042 & 280.36 & 31.59 & 15.01 & 0.54 & 1.34 & 0.06 & 0.56 & 0.03 & 23.3 & 1.2 & $-1.63 \times 10^{-1}$ & $2.03 \times 10^{-2}$ & 45.3 & 7.8 & $-1.38 \times 10^{-1}$ & $1.24 \times 10^{-2}$ \\
\hline & $50-60$ & 0.223 & 0.031 & 350.32 & 21.78 & 16.31 & 0.50 & 1.40 & 0.05 & 0.55 & 0.03 & 25.3 & 4 & $-1.16 \times 10^{-1}$ & $2.48 \times 10^{-2}$ & 54.3 & 4.9 & $-1.38 \times 10^{-1}$ & $1.29 \times 10^{-2}$ \\
\hline & $60-70$ & 0.179 & 0.020 & 407.01 & 14.24 & 17.88 & 0.76 & & & & & 190.0 & 1.8 & & & 44.8 & 2.9 & & \\
\hline & $70-80$ & 0.206 & 0.045 & 341.85 & 25.39 & 16.89 & 0.89 & & & & & 19.3 & 1.5 & & & 47.0 & 3.3 & & \\
\hline & $80-90$ & 0.170 & 0.036 & 380.52 & 30.13 & 18.25 & 0.61 & & & & & & & & & & & & \\
\hline & $90-100$ & 0.173 & 0.045 & 415.47 & 41.74 & 19.07 & 0.44 & & & & & & & & & & & & \\
\hline & $0-10$ & 0.264 & 0.038 & 372.95 & 8.05 & 14.77 & 0.39 & 1.30 & 0.02 & 0.55 & 0.03 & 15.7 & 1.3 & $-3.33 \times 10^{-6}$ & $2.73 \times 10^{-5}$ & 38.7 & 3.4 & $-3.00 \times 10^{-5}$ & $1.73 \times 10^{-5}$ \\
\hline & $10-20$ & 0.293 & 0.026 & 377.08 & 6.43 & 14.93 & 0.21 & 1.30 & NA & 0.55 & NA & 13.0 & NA! & $3.00 \times 10^{-5}$ & NA & 58.0 & NA & $1.20 \times 10^{-4}$ & NA \\
\hline & $20-30$ & 0.234 & 0.025 & 363.45 & 6.87 & 15.37 & 0.26 & & & & & & & & & & & & \\
\hline & $30-40$ & 0.230 & 0.022 & 384.74 & 11.20 & 17.09 & 0.92 & & & & & & & & & & & & \\
\hline 0 & $40-50$ & 0.120 & 0.011 & 434.51 & 15.53 & 22.05 & 0.82 & & & & & & & & & & & & \\
\hline$\infty$ & $50-60$ & 0.073 & 0.006 & 443.61 & 17.93 & 24.65 & 0.58 & 1.36 & 0.02 & 0.48 & 0.02 & 51.5 & 33.8 & $8.25 \times 10^{-5}$ & $2.39 \times 10^{-5}$ & 50.3 & 9.7 & $2.50 \times 10^{-5}$ & $3.12 \times 10^{-5}$ \\
\hline & $60-70$ & 0.084 & 0.005 & 427.79 & 16.50 & 23.79 & 0.66 & & & & & & & & & & & & \\
\hline & $70-80$ & 0.069 & 0.005 & 444.18 & 15.61 & 23.04 & 0.84 & & & & & & & & & & & & \\
\hline & $80-90$ & 0.078 & 0.006 & 457.41 & 19.63 & 23.70 & 1.64 & 1.28 & 0.02 & 0.46 & 0.01 & 19.8 & 3 & $1.70 \times 10^{-4}$ & $2.80 \times 10^{-5}$ & 31.5 & 0.9 & $5.75 \times 10^{-5}$ & $2.56 \times 10^{-5}$ \\
\hline & $90-100$ & 0.086 & 0.012 & 438.05 & 29.43 & 22.10 & 1.17 & & & & & & & & & & & & \\
\hline
\end{tabular}




\begin{tabular}{|c|c|c|c|c|c|c|c|c|c|c|c|c|c|c|c|c|c|c|c|}
\hline \multirow{10}{*}{$\vec{y}$} & $0-10$ & 0.331 & 0.051 & 268.69 & 27.29 & 13.71 & 0.60 & 1.42 & 0.03 & 0.64 & 0.01 & 18.3 & 2 & $7.00 \times 10^{-5}$ & $1.83 \times 10^{-5}$ & 41.5 & 6.9 & $-3.25 \times 10^{-5}$ & $2.72 \times 10^{-5}$ \\
\hline & $10-20$ & 0.450 & 0.025 & 227.14 & 3.19 & 13.77 & 0.34 & & & & & & & & & & & & \\
\hline & $20-30$ & 0.480 & 0.067 & 233.68 & 2.98 & 12.97 & 0.46 & & & & & & & & & & & & \\
\hline & $30-40$ & 0.330 & 0.029 & 298.70 & 24.85 & 13.83 & 0.62 & & & & & & & & & & & & \\
\hline & பी $40-50$ & 0.175 & 0.016 & 409.87 & 11.50 & 15.57 & 0.40 & & & & & & & & & & & & \\
\hline & $50-60$ & 0.130 & 0.021 & 453.43 & 11.09 & 15.02 & 1.14 & 1.24 & 0.03 & 0.54 & 0.03 & 33.8 & 6.8 & $-6.35 \times 10^{-2}$ & $6.34 \times 10^{-5}$ & 55.0 & 8 & $-3.75 \times 10^{-5}$ & $1.93 \times 10^{-5}$ \\
\hline & $60-70$ & 0.132 & 0.007 & 468.77 & 5.30 & 18.04 & 0.94 & & & & & & & & & & & & \\
\hline & $70-80$ & 0.098 & 0.009 & 455.23 & 3.61 & 17.29 & 0.40 & & & & & & & & & & & & \\
\hline & $80-90$ & 0.084 & 0.006 & 474.71 & 2.90 & 18.64 & 0.78 & 1.30 & 0.01 & 0.53 & 0.01 & 21.3 & 2.7 & $1.75 \times 10^{-5}$ & $2.46 \times 10^{-5}$ & 40.3 & 7.7 & $3.75 \times 10^{-5}$ & $1.38 \times 10^{-5}$ \\
\hline & $90-100$ & 0.070 & 0.009 & 456.13 & 8.89 & 19.07 & 1.32 & & & & & & & & & & & & \\
\hline \multirow{9}{*}{ 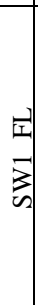 } & $0-10$ & 0.140 & 0.020 & 408.72 & 31.76 & 15.01 & 1.88 & 1.31 & NA & 0.53 & NA & 19.0 & NA & $3.00 \times 10^{-5}$ & NA & 50.0 & NA & $-1.00 \times 10^{-5}$ & NA \\
\hline & $10-20$ & 0.125 & 0.115 & 442.32 & 2.79 & 19.86 & 4.31 & & & & & & & & & & & & \\
\hline & $30-40$ & 0.230 & - & 439.10 & - & 17.44 & - & & & & & & & & & & & & \\
\hline & t. $40-50$ & 0.178 & 0.041 & 381.61 & 52.61 & 18.21 & 0.72 & & & & & & & & & & & & \\
\hline & $50-60$ & 0.290 & 0.041 & 202.73 & 49.97 & 17.49 & 0.98 & 1.80 & 0.19 & 0.92 & 0.12 & 20.5 & 0.5 & $6.00 \times 10^{-5}$ & 0 & 79.0 & 13 & $1.40 \times 10^{-4}$ & $1.00 \times 10^{-5}$ \\
\hline & 60-70 & 0.473 & 0.094 & $-\mathrm{N}$ & - & $-\mathrm{N}$ & - & 1.24 & 0.06 & 0.51 & 0.03 & 27.0 & & $4.00 \times 10^{-5}$ & $9.00 \times 10^{-5}$ & 75.5 & 17.5 & $7.50 \times 10^{-5}$ & $7.50 \times 10^{-5}$ \\
\hline & $70-80$ & 0.160 & 0.039 & 319.95 & 63.89 & 20.03 & 0.89 & & & & & & & & & & & & \\
\hline & $80-90$ & 0.125 & 0.027 & 408.69 & 44.66 & 23.16 & 1.01 & & & & & & & & & & & & \\
\hline & $90-100$ & 0.250 & 0.047 & 402.12 & 34.67 & 21.66 & 0.77 & & & & & & & & & & & & \\
\hline & $0-10$ & 0.163 & 0.018 & 309.04 & 153.11 & 17.21 & 2.67 & 1.30 & NA & 0.47 & NA & 19.0 & NA! & $-5.00 \times 10^{-5}$ & NA & 44.0 & NA & $-9.00 \times 10^{-5}$ & NA \\
\hline & $10-20$ & 0.150 & - & 443.01 & - & 15.88 & - & & & & & & & & & & & & \\
\hline & $30-40$ & 0.107 & 0.047 & 471.47 & 8.50 & 19.56 & 0.93 & & & & & & & & & & & & \\
\hline & 比 40-50 & 0.340 & 0.060 & 305.94 & 72.20 & 18.98 & 1.39 & & & & & & & & & & & & \\
\hline & 기 $50-60$ & 0.230 & 0.081 & 265.11 & 72.40 & 16.62 & 1.17 & 1.35 & NA & 0.47 & NA & 13.0 & NA & $-1.80 \times 10^{-4}$ & NA & 45.0 & NA & $-1.00 \times 10^{-5}$ & NA \\
\hline & 60-70 & 0.185 & 0.054 & 330.40 & 42.95 & 18.35 & 0.94 & 1.58 & NA & 0.65 & NA & 25.0 & NA & $3.00 \times 10^{-5}$ & NA & 65.0 & NA & $5.00 \times 10^{-5}$ & NA \\
\hline & $70-80$ & 0.235 & 0.064 & 339.57 & 47.55 & 21.30 & 1.37 & & & & & & & & & & & & \\
\hline & $80-90$ & 0.223 & 0.059 & 335.91 & 62.95 & 22.04 & 2.33 & 1.26 & 0.05 & 0.51 & 0.02 & 26.0 & 3.0 & $7.00 \times 10^{-5}$ & $2.00 \times 10^{-5}$ & 74.0 & 8.0 & $1.00 \times 10^{-5}$ & $1.00 \times 10^{-5}$ \\
\hline & $90-100$ & 0.465 & 0.016 & 256.62 & 99.18 & 18.07 & 2.57 & & & & & & & & & & & & \\
\hline & $0-10$ & 0.439 & 0.055 & 167.66 & 9.51 & 12.23 & 0.08 & 1.74 & 0.02 & 0.73 & 0.07 & 28.0 & 4.1 & $-4.10 \times 10^{-2}$ & $1.92 \times 10^{-2}$ & 48.3 & 2.0 & $-2.85 \times 10^{-1}$ & $2.89 \times 10^{-2}$ \\
\hline & $10-20$ & 0.568 & 0.016 & 171.26 & 5.17 & 12.25 & 0.12 & & & & & & & & & & & & \\
\hline & $20-30$ & 0.503 & 0.016 & 171.16 & 7.10 & 12.67 & 0.16 & 1.75 & 0.05 & 0.66 & 0.02 & 26.8 & 11.8 & $3.00 \times 10^{-5}$ & $2.64 \times 10^{-2}$ & 47.3 & 6.9 & $-1.01 \times 10^{-1}$ & $3.08 \times 10^{-2}$ \\
\hline & $30-40$ & 0.284 & 0.060 & 321.06 & 51.86 & 15.58 & 0.97 & & & & & & & & & & & & \\
\hline & $40-50$ & 0.324 & 0.025 & 197.43 & 23.33 & 17.37 & 1.06 & & & & & & & & & & & & \\
\hline & 50-60 & 0.194 & 0.024 & 403.12 & 48.27 & 20.98 & 1.47 & 1.38 & 0.04 & 0.52 & 0.11 & 30.3 & 4.2 & $4.91 \times 10^{-2}$ & $3.89 \times 10^{-2}$ & 135 & 35.9 & $2.01 \times 10^{-1}$ & $3.06 \times 10^{-2}$ \\
\hline & $60-70$ & 0.184 & 0.037 & 463.24 & 35.44 & 21.65 & 1.37 & & & & & & & & & & & & \\
\hline & $70-80$ & 0.233 & 0.057 & 407.96 & 55.68 & 20.72 & 1.55 & & & & & & & & & & & & \\
\hline & $80-90$ & 0.188 & 0.038 & 420.09 & 52.61 & 19.89 & 1.45 & & & & & & & & & & & & \\
\hline & $90-100$ & 0.291 & 0.048 & 310.25 & 39.24 & 19.60 & 1.31 & & & & & & & & & & & & \\
\hline & $>100$ & 0.257 & 0.025 & 363.31 & 22.42 & 20.08 & 0.40 & 1.33 & 0.03 & 0.51 & 0.07 & 19.0 & 3 & $2.45 \times 10^{-3}$ & $2.88 \times 10^{-2}$ & 44.5 & 8.5 & $2.79 \times 10^{-1}$ & $4.38 \times 10^{-2}$ \\
\hline \multirow{10}{*}{$\begin{array}{l}0 \\
\Sigma\end{array}$} & $0-10$ & 0.365 & 0.023 & 168.11 & 9.67 & 11.32 & 0.19 & 1.96 & 0.06 & 0.81 & 0.02 & 27.5 & 2.1 & $-1.62 \times 10^{-1}$ & $1.92 \times 10^{-2}$ & 65 & 2.4 & $-2.07 \times 10^{-1}$ & $4.46 \times 10^{-2}$ \\
\hline & $10-20$ & 0.371 & 0.033 & 151.11 & 8.93 & 11.01 & 0.09 & 1.79 & 0.05 & 0.76 & 0.01 & & & & & & & & \\
\hline & $20-30$ & 0.301 & 0.048 & 166.44 & 19.56 & 11.68 & 0.42 & 1.61 & 0.08 & 0.63 & 0.06 & & & & & & & & \\
\hline & $30-40$ & 0.227 & 0.025 & 247.88 & 34.73 & 14.83 & 1.08 & 1.86 & NA & 0.69 & NA & & & & & & & & \\
\hline & 기 $40-50$ & 0.180 & 0.021 & 342.39 & 55.94 & 16.59 & 1.78 & & & & & & & & & & & & \\
\hline & $50-60$ & 0.156 & 0.028 & 388.30 & 30.46 & 18.35 & 0.89 & 1.37 & 0.15 & 0.55 & 0.04 & 29.8 & 7.9 & $1.99 \times 10^{-3}$ & $1.06 \times 10^{-1}$ & 71.8 & 15.3 & $5.87 \times 10^{-2}$ & $4.11 \times 10^{-2}$ \\
\hline & $60-70$ & 0.129 & 0.018 & 461.79 & 9.07 & 19.89 & 0.91 & 1.29 & 0.03 & 0.58 & 0.07 & & & & & & & & \\
\hline & $70-80$ & 0.129 & 0.016 & 457.76 & 14.75 & 19.78 & 0.32 & & & & & & & & & & & & \\
\hline & $80-90$ & 0.184 & 0.031 & 362.97 & 56.72 & 20.19 & 0.94 & 1.46 & 0.08 & 0.61 & 0.07 & 23.5 & 2.6 & $2.36 \times 10^{-1}$ & $1.45 \times 10^{-1}$ & 52.7 & 10.5 & $1.95 \times 10^{-1}$ & $3.21 \times 10^{-2}$ \\
\hline & $90-100$ & 0.125 & 0.007 & 444.22 & 19.05 & 19.77 & 0.63 & & & & & & & & & & & & \\
\hline \multirow{10}{*}{ 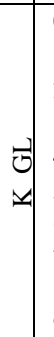 } & $0-10$ & 0.390 & 0.112 & 229.89 & 7.43 & 11.81 & 0.14 & 1.73 & 0.03 & 0.76 & 0.03 & 29.8 & 4.2 & $-1.41 \times 10^{-1}$ & $4.46 \times 10^{-2}$ & 60.3 & 6.6 & $-2.33 \times 10^{-1}$ & $4.07 \times 10^{-2}$ \\
\hline & $10-20$ & 0.323 & 0.027 & 213.98 & 7.51 & 12.10 & 0.18 & 1.64 & 0.04 & 0.67 & 0.06 & & & & & & & & \\
\hline & $20-30$ & 0.265 & 0.024 & 212.60 & 11.12 & 12.17 & 0.22 & 1.59 & 0.05 & 0.70 & 0.05 & & & & & & & & \\
\hline & $30-40$ & 0.183 & 0.015 & 330.26 & 39.38 & 16.47 & 1.25 & & & & & & & & & & & & \\
\hline & t) $40-50$ & 0.239 & 0.040 & 351.68 & 46.69 & 15.81 & 1.30 & & & & & & & & & & & & \\
\hline & $50-60$ & 0.159 & 0.022 & 400.21 & 27.44 & 17.37 & 1.01 & 1.31 & 0.03 & 0.63 & 0.05 & 19.0 & 3.7 & $-8.53 \times 10^{-2}$ & $6.95 \times 10^{-2}$ & 54.8 & 6.9 & $1.02 \times 10^{-1}$ & $1.01 \times 10^{-1}$ \\
\hline & $60-70$ & 0.183 & 0.028 & 324.97 & 47.17 & 15.49 & 0.41 & 1.54 & 0.15 & 0.65 & 0.19 & & & & & & & & \\
\hline & $70-80$ & 0.140 & 0.019 & 390.82 & 34.48 & 17.38 & 0.37 & & & & & & & & & & & & \\
\hline & $80-90$ & 0.119 & 0.008 & 428.71 & 16.85 & 19.57 & 0.66 & 1.32 & 0.03 & 0.62 & 0.07 & 41.8 & 14.2 & $4.26 \times 10^{-1}$ & $1.09 \times 10^{-1}$ & 70.8 & 27.2 & $3.16 \times 10^{-1}$ & $8.28 \times 10^{-2}$ \\
\hline & 90-100 & 0.131 & 0.009 & 394.83 & 10.96 & 18.19 & 0.73 & & & & & & & & & & & & \\
\hline
\end{tabular}




\begin{tabular}{|c|c|c|c|c|c|c|c|c|c|c|c|c|c|c|c|c|c|c|c|}
\hline \multirow{10}{*}{ 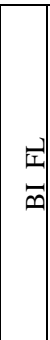 } & $0-10$ & 0.163 & 0.047 & 467.21 & 4.91 & 20.46 & 0.47 & \multirow{4}{*}{1.38} & \multirow{4}{*}{0.04} & \multirow{4}{*}{0.44} & \multirow{4}{*}{0.06} & \multirow{4}{*}{27.3} & \multirow[b]{3}{*}{5} & \multirow[b]{3}{*}{$2.50 \times 10^{-6}$} & \multirow{4}{*}{$6.03 \times 10^{-5}$} & \multirow{4}{*}{103.3} & \multirow{4}{*}{23.7} & \multirow{4}{*}{$5.25 \times 10^{-5}$} & \multirow[b]{3}{*}{$4.48 \times 10^{-5}$} \\
\hline & $10-20$ & 0.126 & 0.022 & 461.57 & 7.45 & 22.13 & 0.81 & & & & & & & & & & & & \\
\hline & $20-30$ & 0.169 & 0.019 & 477.77 & 14.76 & 22.83 & 0.62 & & & & & & & & & & & & \\
\hline & $30-40$ & 0.155 & 0.004 & 491.70 & 8.69 & 23.48 & 0.61 & & & & & & & & & & & & \\
\hline & $40-50$ & 0.144 & 0.004 & 495.86 & 8.81 & 23.15 & 0.47 & & & & & & & & & & & & \\
\hline & $50-60$ & 0.174 & 0.021 & 435.63 & 17.78 & 25.06 & 1.73 & 1.26 & 0.03 & 0.44 & 0.02 & 14.3 & 2.8 & $-9.25 \times 10^{-5}$ & $2.93 \times 10^{-5}$ & 42.8 & 6.1 & $6.25 \times 10^{-5}$ & $2.39 \times 10^{-5}$ \\
\hline & $60-70$ & 0.290 & 0.022 & 424.76 & 19.40 & 33.26 & 4.92 & & & & & & & & & & & & \\
\hline & $70-80$ & 0.328 & 0.036 & 377.24 & 29.61 & 31.73 & 3.94 & & & & & & & & & & & & \\
\hline & $80-90$ & 0.431 & 0.130 & 241.07 & 62.19 & 20.45 & 3.95 & 1.49 & 0.08 & 0.50 & 0.06 & 20.8 & 7.4 & $-5.00 \times 10^{-5}$ & $7.22 \times 10^{-5}$ & 49.8 & 8.5 & $1.35 \times 10^{-4}$ & $2.02 \times 10^{-5}$ \\
\hline & $90-100$ & 0.474 & 0.106 & 205.08 & 52.08 & 21.42 & 1.06 & & & & & & & & & & & & \\
\hline & $0-10$ & 0.034 & 0.005 & 384.61 & 33.31 & 16.05 & 1.54 & 1.44 & 0.08 & 0.53 & 0.03 & 49.3 & 11.7 & $6.03 \times 10^{-2}$ & $3.17 \times 10^{-2}$ & 93.5 & 17 & $-7.71 \times 10^{-2}$ & $3.26 \times 10^{-2}$ \\
\hline & $10-20$ & 0.054 & 0.015 & 402.39 & 53.40 & 17.70 & 0.76 & & & & & 26.8 & 11.8 & & & 47.3 & 6.9 & & \\
\hline & $20-30$ & 0.058 & 0.012 & 394.58 & 46.98 & 15.26 & 0.38 & & & & & & & & & & & & \\
\hline & $30-40$ & 0.039 & 0.003 & 454.17 & 16.98 & 15.93 & 0.82 & & & & & & & & & & & & \\
\hline & $40-50$ & 0.035 & 0.008 & 415.39 & 42.37 & 16.01 & 1.61 & & & & & & & & & & & & \\
\hline - & $50-60$ & 0.031 & 0.005 & 463.89 & 12.23 & 18.77 & 0.78 & 1.32 & 0.02 & 0.52 & 0.02 & 33.3 & 3.2 & $8.63 \times 10^{-2}$ & $2.73 \times 10^{-2}$ & 126.3 & 27.7 & $1.90 \times 10^{-1}$ & $9.88 \times 10^{-2}$ \\
\hline 7 & $60-70$ & 0.033 & 0.003 & 470.39 & 10.35 & 21.83 & 1.20 & & & & & & & & & & & & \\
\hline 3 & $70-80$ & 0.036 & 0.003 & 466.53 & 15.62 & 19.61 & 0.97 & & & & & & & & & & & & \\
\hline & $80-90$ & 0.026 & 0.006 & 449.25 & 20.30 & 18.72 & 1.85 & 1.39 & 0.04 & 0.52 & 0.01 & 40.3 & 2.6 & $2.40 \times 10^{-1}$ & $6.12 \times 10^{-2}$ & 288 & 50.6 & $7.12 \times 10^{-1}$ & $9.60 \times 10^{-2}$ \\
\hline & $90-100$ & 0.034 & 0.007 & 432.21 & 27.96 & 16.89 & 0.42 & & & & & & & & & & & & \\
\hline & $0-10$ & 0.020 & 0.004 & 472.03 & 7.38 & 21.69 & 1.55 & 1.41 & 0.02 & 0.51 & 0.03 & 59.0 & 5.5 & $2.27 \times 10^{-2}$ & $3.07 \times 10^{-2}$ & 151 & 19.6 & $5.20 \times 10^{-3}$ & $2.49 \times 10^{-2}$ \\
\hline & $10-20$ & 0.031 & 0.004 & 460.82 & 4.55 & 19.59 & 0.42 & 1.45 & NA & 0.45 & NA & 17.0 & NA & $8.13 \times 10^{-2}$ & NA & 38 & NA & $6.93 \times 10^{-2}$ & NA \\
\hline & $20-30$ & 0.035 & 0.003 & 493.02 & 9.04 & 22.87 & 1.69 & & & & & & & & & & & & \\
\hline & $30-40$ & 0.040 & 0.003 & 478.59 & 16.91 & 20.74 & 1.02 & & & & & & & & & & & & \\
\hline 国 & $40-50$ & 0.035 & 0.004 & 476.88 & 13.73 & 19.61 & 1.29 & & & & & & & & & & & & \\
\hline 3 & $50-60$ & 0.040 & 0.005 & 482.76 & 14.02 & 21.44 & 1.55 & 1.39 & 0.01 & 0.46 & 0.02 & 30.0 & 4.2 & $2.27 \times 10^{-1}$ & $5.74 \times 10^{-2}$ & 61.3 & 3 & $1.02 \times 10^{-1}$ & $7.06 \times 10^{-2}$ \\
\hline & $60-70$ & 0.053 & 0.009 & 471.48 & 24.22 & 30.63 & 5.98 & & & & & & & & & & & & \\
\hline & $70-80$ & 0.073 & 0.024 & 412.13 & 53.84 & 27.24 & 5.60 & & & & & & & & & & & & \\
\hline & $80-90$ & 0.040 & 0.008 & 396.55 & 68.94 & 21.98 & 3.61 & 1.40 & 0.02 & 0.49 & 0.02 & 26.3 & 1.8 & $1.23 \times 10^{-1}$ & $1.02 \times 10^{-1}$ & 65 & 12.7 & $9.38 \times 10^{-2}$ & $4.11 \times 10^{-2}$ \\
\hline & $90-100$ & 0.152 & 0.079 & 457.31 & 24.59 & 16.89 & 2.30 & & & & & & & & & & & & \\
\hline & $0-10$ & 0.058 & 0.008 & 427.64 & 23.16 & 20.93 & 2.26 & 1.61 & 0.04 & 0.54 & 0.11 & 99.3 & 32.2 & $7.39 \times 10^{-2}$ & $1.20 \times 10^{-1}$ & 172.3 & 40.8 & $-2.75 \times 10^{-1}$ & $2.37 \times 10^{-1}$ \\
\hline & $10-20$ & 0.157 & 0.040 & 425.64 & 14.49 & 15.46 & 0.51 & 1.21 & 0.21 & 0.44 & 0.10 & & & & & & & & \\
\hline & $20-30$ & 0.194 & 0.018 & 400.90 & 22.53 & 15.53 & 0.78 & 1.53 & 0.03 & 0.45 & 0.03 & & & & & & & & \\
\hline & $30-40$ & 0.160 & 0.008 & 424.79 & 25.08 & 17.61 & 0.49 & & & & & & & & & & & & \\
\hline ज) & $40-50$ & 0.110 & 0.014 & 432.72 & 18.52 & 17.48 & 0.59 & & & & & & & & & & & & \\
\hline$=$ & $50-60$ & 0.210 & 0.022 & 389.91 & 25.43 & 18.18 & 0.64 & 1.52 & 0.04 & 0.44 & 0.05 & 41.0 & 10.5 & $-2.59 \times 10^{-1}$ & $1.20 \times 10^{-1}$ & 96 & 22.3 & $3.47 \times 10^{-1}$ & $1.44 \times 10^{-1}$ \\
\hline & $60-70$ & 0.190 & 0.029 & 426.93 & 21.54 & 24.31 & 4.52 & 1.44 & 0.07 & 0.53 & 0.04 & & & & & & & & \\
\hline & $70-80$ & 0.240 & 0.040 & 342.47 & 46.46 & 20.17 & 1.77 & & & & & & & & & & & & \\
\hline & $80-90$ & 0.235 & 0.042 & 327.91 & 31.93 & 22.40 & 3.76 & 1.47 & 0.02 & 0.62 & 0.05 & 32.5 & 8.8 & $9.28 \times 10^{-2}$ & $2.12 \times 10^{-1}$ & 82 & 8.5 & $6.03 \times 10^{-2}$ & $1.65 \times 10^{-1}$ \\
\hline & $90-100$ & 0.186 & 0.029 & 334.23 & 31.98 & 21.69 & 2.82 & & & & & & & & & & & & \\
\hline & $0-10$ & 0.140 & 0.035 & 404.06 & 42.40 & 19.80 & 1.01 & 1.48 & 0.07 & 0.61 & 0.03 & 65.8 & 16.4 & $-7.14 \times 10^{-2}$ & $8.35 \times 10^{-2}$ & 158.3 & 54.1 & $-1.96 \times 10^{-1}$ & $1.14 \times 10^{-1}$ \\
\hline & $10-20$ & 0.106 & 0.019 & 437.95 & 12.08 & 19.51 & 2.43 & 1.43 & 0.17 & 0.51 & 0.05 & & & & & & & & \\
\hline & $20-30$ & 0.193 & 0.026 & 417.53 & 15.42 & 18.12 & 0.72 & 1.49 & 0.05 & 0.42 & 0.07 & & & & & & & & \\
\hline & $30-40$ & 0.171 & 0.014 & 403.32 & 31.45 & 16.88 & 0.41 & & & & & & & & & & & & \\
\hline 国 & $40-50$ & 0.158 & 0.016 & 426.19 & 33.27 & 17.42 & 0.81 & & & & & & & & & & & & \\
\hline 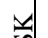 & $50-60$ & 0.173 & 0.035 & 449.63 & 33.95 & 18.19 & 0.65 & 1.41 & 0.07 & 0.42 & 0.10 & 35.3 & 8.4 & $9.00 \times 10^{-3}$ & $1.24 \times 10^{-1}$ & 80.5 & 21.9 & $-2.26 \times 10^{-1}$ & $1.06 \times 10^{-1}$ \\
\hline & $60-70$ & 0.153 & 0.031 & 473.91 & 5.39 & 21.83 & 1.99 & 1.40 & 0.02 & 0.50 & 0.04 & & & & & & & & \\
\hline & $70-80$ & 0.137 & 0.009 & 418.65 & 40.17 & 17.64 & 0.12 & & & & & & & & & & & & \\
\hline & $80-90$ & 0.145 & 0.013 & 413.58 & 47.33 & 19.67 & 2.15 & 1.49 & 0.05 & 0.52 & 0.11 & 52.0 & NA & $6.04 \times 10^{-1}$ & NA & 122 & NA & $5.20 \times 10^{-1}$ & NA \\
\hline & $90-100$ & 0.133 & 0.024 & 389.36 & 77.94 & 20.57 & 2.76 & & & & & & & & & & & & \\
\hline
\end{tabular}




\begin{tabular}{|c|c|c|c|c|c|c|c|c|c|c|c|c|c|c|c|c|c|c|c|}
\hline \multirow{10}{*}{ (4) } & $0-10$ & 0.198 & 0.031 & 202.07 & 7.70 & 12.54 & 0.16 & 1.72 & 0.01 & 0.69 & 0.02 & 57.5 & 5.3 & $7.31 \times 10^{-3}$ & $7.22 \times 10^{-2}$ & 109.5 & 6 & $-1.79 \times 10^{-1}$ & $1.68 \times 10^{-1}$ \\
\hline & $10-20$ & 0.340 & 0.025 & 158.78 & 2.94 & 14.02 & 0.34 & 1.74 & 0.05 & 0.97 & 0.28 & 25.0 & 2.9 & $-2.69 \times 10^{-1}$ & $7.75 \times 10^{-2}$ & 62.3 & 5 & $-2.76 \times 10^{-1}$ & $8.49 \times 10^{-2}$ \\
\hline & $20-30$ & 0.221 & 0.034 & 191.12 & 12.90 & 15.87 & 0.55 & 1.63 & 0.05 & 0.79 & 0.18 & 29.7 & 1.5 & $-4.29 \times 10^{-1}$ & $9.72 \times 10^{-2}$ & 49.7 & 4.9 & $-4.19 \times 10^{-1}$ & $6.76 \times 10^{-2}$ \\
\hline & $30-40$ & 0.117 & 0.014 & 293.57 & 37.71 & 19.55 & 1.24 & 1.34 & NA & 0.23 & NA & 6.0 & NA & $-1.30 \times 10^{-1}$ & NA & 18 & NA & $-2.94 \times 10^{-1}$ & NA \\
\hline & $40-50$ & 0.064 & 0.006 & 415.86 & 9.34 & 25.03 & 1.08 & & & & & & & & & & & & \\
\hline & $50-60$ & 0.116 & 0.016 & 435.56 & 15.39 & 24.88 & 0.92 & 1.34 & 0.00 & 0.46 & 0.03 & 17.5 & 2.2 & $1.41 \times 10^{-1}$ & $1.21 \times 10^{-1}$ & 80.3 & 7.6 & $6.33 \times 10^{-1}$ & $1.41 \times 10^{-1}$ \\
\hline & $60-70$ & 0.195 & 0.039 & 223.71 & 52.18 & 25.75 & 1.97 & 1.69 & 0.15 & 1.00 & 0.33 & 32.5 & 2.9 & $4.67 \times 10^{-1}$ & $1.16 \times 10^{-1}$ & 53.8 & 12.6 & $1.17 \times 10^{-1}$ & $8.14 \times 10^{-2}$ \\
\hline & $70-80$ & 0.112 & 0.010 & 306.50 & 36.01 & 22.45 & 0.52 & & & & & & & & & & & & \\
\hline & $80-90$ & 0.144 & 0.016 & 331.09 & 27.51 & 25.68 & 1.14 & 1.39 & 0.04 & 0.51 & 0.02 & 28.7 & 1.8 & $4.31 \times 10^{-1}$ & $9.20 \times 10^{-2}$ & 83.0 & 14 & $3.28 \times 10^{-1}$ & $1.19 \times 10^{-1}$ \\
\hline & $90-100$ & 0.118 & 0.016 & 405.12 & 20.83 & 26.30 & 0.46 & 1.66 & NA & 0.69 & NA & 53.0 & NA & $4.42 \times 10^{-1}$ & NA & 58.0 & NA & $2.81 \times 10^{-1}$ & NA \\
\hline \multirow{10}{*}{ 니 } & $0-10$ & 0.493 & 0.145 & 150.27 & 34.24 & 12.95 & 1.40 & 1.78 & 0.18 & 0.84 & 0.05 & 39.3 & 4.9 & $-1.00 \times 10^{-4}$ & $3.00 \times 10^{-5}$ & 84.3 & 16 & $2.33 \times 10^{-5}$ & $7.42 \times 10^{-5}$ \\
\hline & $10-20$ & 0.576 & 0.105 & 118.71 & 13.25 & 12.26 & 0.44 & 1.92 & NA & 0.87 & NA & 156 & NA & $-2.20 \times 10^{-4}$ & NA & 260.0 & NA & $8.00 \times 10^{-5}$ & NA \\
\hline & $20-30$ & 0.518 & 0.042 & 142.24 & 13.90 & 12.27 & 0.46 & & & & & & & & & & & & \\
\hline & $30-40$ & 0.259 & 0.036 & 291.98 & 32.99 & 17.57 & 1.30 & & & & & & & & & & & & \\
\hline & $40-50$ & 0.154 & 0.014 & 359.54 & 22.03 & 20.13 & 0.44 & & & & & & & & & & & & \\
\hline & $50-60$ & 0.181 & 0.037 & 351.69 & 20.76 & 22.36 & 1.20 & 1.39 & 0.05 & 0.59 & 0.04 & 44.8 & 12.8 & $3.50 \times 10^{-5}$ & $6.33 \times 10^{-5}$ & 135.3 & 7.6 & $-7.75 \times 10^{-5}$ & $6.05 \times 10^{-5}$ \\
\hline & $60-70$ & 0.219 & 0.025 & 319.68 & 33.16 & 22.50 & 1.17 & & & & & & & & & & & & \\
\hline & $70-80$ & 0.189 & 0.027 & 301.22 & 39.86 & 22.34 & 1.36 & & & & & & & & & & & & \\
\hline & $80-90$ & 0.184 & 0.029 & 252.77 & 33.42 & 20.51 & 0.68 & 1.50 & 0.07 & 0.62 & 0.03 & 93.5 & 27.6 & $-7.50 \times 10^{-6}$ & $4.09 \times 10^{-5}$ & 125.3 & 11.7 & $-1.25 \times 10^{-5}$ & $1.11 \times 10^{-5}$ \\
\hline & $90-100$ & 0.160 & 0.017 & 318.05 & 25.00 & 22.16 & 0.86 & & & & & & & & & & & & \\
\hline \multirow{10}{*}{$\underline{\Xi}$} & $0-10$ & 0.496 & 0.061 & 101.13 & 4.24 & 10.92 & 0.55 & 2.17 & 0.09 & 1.08 & 0.04 & 37.3 & 6.2 & $-7.75 \times 10^{-5}$ & $5.17 \times 10^{-5}$ & 61.8 & 3 & $-1.25 \times 10^{-5}$ & $4.82 \times 10^{-5}$ \\
\hline & $10-20$ & 0.599 & 0.029 & 98.77 & 6.59 & 10.85 & 0.51 & & & & & & & & & & & & \\
\hline & $20-30$ & 0.544 & 0.078 & 99.26 & 5.99 & 10.91 & 0.32 & & & & & & & & & & & & \\
\hline & $30-40$ & 0.404 & 0.071 & 118.60 & 19.66 & 11.55 & 0.78 & & & & & & & & & & & & \\
\hline & $40-50$ & 0.290 & 0.048 & 165.26 & 17.43 & 12.75 & 0.82 & & & & & & & & & & & & \\
\hline & $50-60$ & 0.235 & 0.052 & 245.78 & 29.82 & 16.81 & 1.01 & 1.47 & 0.08 & 0.63 & 0.06 & 35.8 & 9.3 & $-3.00 \times 10^{-5}$ & $3.76 \times 10^{-5}$ & 106.3 & 33.8 & $-3.50 \times 10^{-5}$ & $5.44 \times 10^{-5}$ \\
\hline & $60-70$ & 0.196 & 0.023 & 319.98 & 37.96 & 19.30 & 0.83 & & & & & & & & & & & & \\
\hline & $70-80$ & 0.164 & 0.014 & 352.24 & 32.78 & 19.64 & 0.56 & & & & & & & & & & & & \\
\hline & $80-90$ & 0.154 & 0.014 & 344.57 & 24.12 & 21.31 & 1.00 & 1.33 & 0.03 & 0.51 & 0.06 & 41.5 & 6 & $4.50 \times 10^{-5}$ & $6.45 \times 10^{-6}$ & 99.3 & 14.3 & $4.25 \times 10^{-5}$ & $1.31 \times 10^{-5}$ \\
\hline & $90-100$ & 0.145 & 0.005 & 368.36 & 18.17 & 21.14 & 0.68 & & & & & & & & & & & & \\
\hline \multirow{10}{*}{$\begin{array}{l}\text { 国 } \\
x\end{array}$} & $0-10$ & 0.081 & 0.013 & 461.39 & 13.17 & 23.97 & 1.49 & 1.29 & 0.01 & 0.49 & 0.01 & 26.5 & 3.8 & $-7.00 \times 10^{-5}$ & $2.08 \times 10^{-5}$ & 76.8 & 8.2 & $-1.25 \times 10^{-5}$ & $1.25 \times 10^{-5}$ \\
\hline & $10-20$ & 0.129 & 0.015 & 454.53 & 9.90 & 18.22 & 1.27 & & & & & & & & & & & & \\
\hline & $20-30$ & 0.140 & 0.009 & 449.79 & 7.86 & 16.03 & 0.99 & & & & & & & & & & & & \\
\hline & $30-40$ & 0.136 & 0.004 & 467.86 & 6.70 & 18.62 & 0.78 & & & & & & & & & & & & \\
\hline & $40-50$ & 0.144 & 0.004 & 469.67 & 5.83 & 19.97 & 0.58 & & & & & & & & & & & & \\
\hline & $50-60$ & 0.138 & 0.009 & 469.07 & 3.14 & 20.93 & 1.22 & 1.26 & 0.02 & 0.50 & 0.00 & 20.5 & 3.6 & $-3.50 \times 10^{-5}$ & $4.17 \times 10^{-5}$ & 59.3 & 5.5 & $-7.50 \times 10^{-6}$ & $3.09 \times 10^{-5}$ \\
\hline & $60-70$ & 0.134 & 0.006 & 472.80 & 4.18 & 20.70 & 0.49 & 1.26 & 0.03 & 0.50 & 0.01 & 20.5 & 2.5 & $-8.00 \times 10^{-5}$ & $1.00 \times 10^{-5}$ & 48.0 & 1 & $-5.00 \times 10^{-6}$ & $5.00 \times 10^{-6}$ \\
\hline & $70-80$ & 0.227 & 0.056 & 470.67 & 9.06 & 20.37 & 1.65 & & & & & & & & & & & & \\
\hline & $80-90$ & 0.118 & 0.010 & 471.11 & 11.44 & 18.56 & 1.89 & 1.34 & 0.09 & 0.49 & 0.03 & 37.0 & 16.0 & $-2.50 \times 10^{-5}$ & $5.50 \times 10^{-5}$ & 45.0 & 6 & $-6.00 \times 10^{-5}$ & $2.00 \times 10^{-5}$ \\
\hline & $90-100$ & 0.295 & 0.106 & 489.43 & 4.79 & 21.40 & 1.80 & & & & & & & & & & & & \\
\hline \multirow{10}{*}{$\begin{array}{l}\text { 国 } \\
\text { II }\end{array}$} & $0-10$ & 0.134 & 0.012 & 442.36 & 8.32 & 14.38 & 0.41 & 1.37 & 0.02 & 0.47 & 0.01 & 51.3 & 7.6 & $-4.75 \times 10^{-5}$ & $2.59 \times 10^{-5}$ & 89.8 & 8 & $-4.00 \times 10^{-5}$ & $6.18 \times 10^{-5}$ \\
\hline & $10-20$ & 0.249 & 0.065 & 404.17 & 14.54 & 13.76 & 0.53 & & & & & & & & & & & & \\
\hline & $20-30$ & 0.208 & 0.009 & 459.37 & 18.26 & 16.58 & 0.89 & & & & & & & & & & & & \\
\hline & $30-40$ & 0.188 & 0.020 & 440.63 & 10.21 & 17.02 & 0.74 & & & & & & & & & & & & \\
\hline & $40-50$ & 0.131 & 0.005 & 479.59 & 6.10 & 18.25 & 0.65 & & & & & & & & & & & & \\
\hline & $50-60$ & 0.133 & 0.009 & 492.31 & 7.86 & 17.52 & 0.53 & 1.31 & 0.01 & 0.43 & 0.01 & 18.3 & 2.9 & $9.00 \times 10^{-5}$ & $6.75 \times 10^{-5}$ & 48.5 & 9.4 & $4.75 \times 10^{-5}$ & $5.51 \times 10^{-5}$ \\
\hline & $60-70$ & 0.161 & 0.005 & 499.16 & 8.07 & 18.32 & 0.37 & & & & & & & & & & & & \\
\hline & $70-80$ & 0.178 & 0.007 & 475.44 & 8.06 & 20.06 & 0.45 & & & & & & & & & & & & \\
\hline & $80-90$ & 0.181 & 0.012 & 482.42 & 7.31 & 21.05 & 0.87 & 1.21 & 0.02 & 0.45 & 0.01 & 19.5 & 3.1 & $-1.75 \times 10^{-5}$ & $7.27 \times 10^{-5}$ & 0.0985 & 0.0360 & $1.90 \times 10^{-4}$ & $5.64 \times 10^{-5}$ \\
\hline & $90-100$ & 0.180 & 0.008 & 488.60 & 5.18 & 23.07 & 0.54 & & & & & & & & & & & & \\
\hline
\end{tabular}

